\title{
Tau filaments from multiple cases of sporadic and inherited Alzheimer's disease adopt a common fold
}

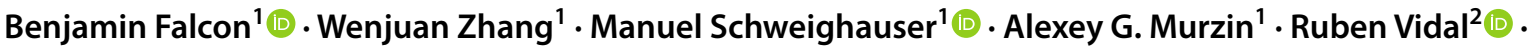

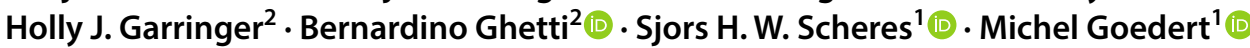

Received: 21 August 2018 / Revised: 20 September 2018 / Accepted: 20 September 2018 / Published online: 1 October 2018

(c) The Author(s) 2018

\begin{abstract}
The ordered assembly of tau protein into abnormal filaments is a defining characteristic of Alzheimer's disease (AD) and other neurodegenerative disorders. It is not known if the structures of tau filaments vary within, or between, the brains of individuals with $\mathrm{AD}$. We used a combination of electron cryo-microscopy (cryo-EM) and immuno-gold negative-stain electron microscopy (immuno-EM) to determine the structures of paired helical filaments (PHFs) and straight filaments (SFs) from the frontal cortex of 17 cases of $\mathrm{AD}$ (15 sporadic and 2 inherited) and 2 cases of atypical AD (posterior cortical atrophy). The high-resolution structures of PHFs and SFs from the frontal cortex of 3 cases of AD, 2 sporadic and 1 inherited, were determined by cryo-EM. We also used immuno-EM to study the PHFs and SFs from a number of cortical and subcortical brain regions. PHFs outnumbered SFs in all AD cases. By cryo-EM, PHFs and SFs were made of two C-shaped protofilaments with a combined cross- $\beta / \beta$-helix structure, as described previously for one case of $\mathrm{AD}$. The higher resolution structures obtained here showed two additional amino acids at each end of the protofilament. The immuno-EM findings, which indicated the presence of repeats 3 and 4, but not of the N-terminal regions of repeats 1 and 2, of tau in the filament cores of all $\mathrm{AD}$ cases, were consistent with the cryo-EM results. These findings show that there is no significant variation in tau filament structures between individuals with AD. This knowledge will be crucial for understanding the mechanisms that underlie tau filament formation and for developing novel diagnostics and therapies.
\end{abstract}

Keywords Alzheimer's disease · Neurodegenerative diseases · Tau protein · Paired helical filaments · Straight filaments · Electron cryo-microscopy $\cdot$ Immuno-gold negative-stain electron microscopy

Benjamin Falcon and Wenjuan Zhang contributed equally to this work.

Sjors H. W. Scheres and Michel Goedert jointly supervised this work.

Electronic supplementary material The online version of this article (https://doi.org/10.1007/s00401-018-1914-z) contains supplementary material, which is available to authorized users.

Sjors H. W. Scheres

scheres@mrc-lmb.cam.ac.uk

$\triangle$ Michel Goedert

mg@mrc-lmb.cam.ac.uk

1 MRC Laboratory of Molecular Biology, Cambridge CB2 0QH, UK

2 Department of Pathology and Laboratory Medicine, Indiana University School of Medicine, Indianapolis, IN 46202, USA

\section{Introduction}

Alzheimer's disease (AD) is the most common neurodegenerative disorder [33]. The vast majority of cases are sporadic, with age being the main predisposing factor. Inheritance of the $\varepsilon 4$ allele of the apolipoprotein $\mathrm{E}$ gene $(A P O E)$ is a major genetic risk factor for sporadic AD [37]. Dominantly inherited forms of $\mathrm{AD}$, which are caused by mutations in the amyloid precursor protein gene $(A P P)[17,29]$ and the presenilin genes (PSEN1 and PSEN2) [26, 35], account for less than $1 \%$ of cases. No mechanism-based therapies exist.

All cases of AD are characterised by abundant intraneuronal neurofibrillary lesions of filamentous tau $[4,22,41$, $42]$ and extracellular deposits of filamentous $\beta$-amyloid (A $\beta)[16,27]$ in the cerebral cortex and other brain regions. Atypical forms of AD differ from typical forms by clinical presentation and the brain areas most severely affected by neurofibrillary lesions and $A \beta$ deposits [15]. Despite being 
made of unrelated proteins, tau and $A \beta$ filaments share a cross- $\beta$ architecture characteristic of amyloids $[2,16]$. Filamentous tau inclusions, in the absence of $\mathrm{A} \beta$ deposits, define a number of other neurodegenerative diseases [18]. Six tau isoforms are expressed in normal adult human brain: three isoforms have four microtubule-binding repeats $(\mathrm{R} 1, \mathrm{R} 2, \mathrm{R} 3$, $\mathrm{R} 4 ; 4 \mathrm{R}$ tau) and three isoforms lack the second repeat (3R tau). Tau filaments of AD are composed of all six isoforms [20].

Neurofibrillary lesions consist of tangles in cell bodies, neuropil threads in the processes of nerve cells, and dystrophic neurites associated with plaques. The accumulation of neurofibrillary lesions in brain follows a stereotypical pattern that correlates with atrophy and cognitive deficits [3]. It follows that an understanding of the ordered assembly of tau into filaments is essential for diagnosis and therapy. Tau filaments from AD brain adopt two characteristic morphologies. Paired helical filaments (PHFs) constitute the major species; they have a helical crossover distance of approximately $70 \mathrm{~nm}$, with pronounced variations in projected widths from 7 to $15 \mathrm{~nm}$. Straight filaments (SFs), which have a similar crossover distance, but a constant width of approximately $10 \mathrm{~nm}$, are in the minority [8].

The structures of PHFs and SFs extracted from the frontal cortex of an individual with sporadic AD were previously determined using electron cryo-microscopy (cryo-EM) [14]. PHFs and SFs were found to be ultrastructural polymorphs, which share two identical protofilaments, but differ in their inter-protofilament packing. Each $\mathrm{C}$-shaped protofilament adopted a combined cross- $\beta / \beta$-helix structure comprising residues 306-378 of human tau (in the numbering of the 441 residue isoform), i.e., R3, R4, and 10 amino acids following R4. Cryo-EM was also used to determine the structures of tau filaments from the frontotemporal cortex of an individual with Pick's disease (PiD) [12], a frontotemporal dementia with tau filaments made of $3 \mathrm{R}$ tau. The ordered core of tau filaments from PiD was made of a single protofilament with an elongated cross- $\beta$ structure, comprising residues K254-F378 of tau (but lacking V275-S305 of R2). Distinct conformers of aggregated tau thus exist in human tauopathies.

It is not known if the structures of tau filaments vary within, or between, the brains of individuals with $\mathrm{AD}$. This knowledge is vital for the development of therapies targeting tau assembly and the design of diagnostic ligands. It is also essential for understanding the mechanisms underlying tau filament formation and propagation.

Here, we used cryo-EM to determine the structures of tau filaments from two additional cases of sporadic AD and one case of dominantly inherited disease (V717F mutation in $A P P)$. Since cryo-EM is too low throughput to study a large number of cases, we also used immuno-gold negative-stain electron microscopy (immuno-EM) to determine the morphologies and tau repeat composition of the core of PHFs and SFs from frontal cortex of the four cases used for cryo-EM, an additional 12 cases with the typical form of sporadic $\mathrm{AD}$, and one more case with dominantly inherited disease. We also used immuno-EM to study tau filaments from temporal, occipital, and cingulate cortices, as well as from thalamus, substantia innominata, and putamen of $\mathrm{AD}$ case 1 used previously for cryo-EM [14]. Immuno-EM was performed as well on tau fillaments extracted from frontal and occipital cortices of two cases of posterior cortical atrophy (PCA). PCA is an atypical form of sporadic AD with the early visual dysfunction and neurodegeneration of posterior cortical regions $[1,9]$. Our immuno-EM results are consistent with the core of PHFs and SFs from all AD cases and brain regions examined comprising R3 and R4 of tau, while our cryo-EM structures demonstrate that PHFs and SFs from frontal cortex of the three new cases of AD are like those we previously reported [14], indicating the existence of a common tau fold in AD.

\section{Materials and methods}

\section{Extraction of tau filaments}

Sporadic cases of typical AD were selected according to several criteria, including a wide spread in the ages at death of patients and in the post-mortem intervals (time between death and brain autopsy), a tau pathology of Braak stage 6 , and no or only mild co-pathologies. Inherited cases of typical AD were chosen based on the presence of a V717F mutation in APP and sporadic cases of atypical AD exhibited a clinical and neuropathological picture of PCA. Neurohistology and immunohistochemistry were carried out as described [36].

Sarkosyl-insoluble material was extracted according to [20]. Approximately $6 \mathrm{~g}$ frontal cortex was used for cryo-EM and $0.6 \mathrm{~g}$ from each brain region studied for immuno-EM. The pelleted sarkosyl-insoluble material was resuspended in $10 \mathrm{mM}$ Tris-HCl pH 7.4, $800 \mathrm{mM} \mathrm{NaCl}, 5 \mathrm{mM}$ EDTA, and $1 \mathrm{mM}$ EGTA, with a final concentration of $10 \%(\mathrm{w} / \mathrm{v})$ sucrose at $750 \mu \mathrm{l}$ per $\mathrm{g}$ tissue, followed by centrifugation at $20,100 \mathrm{~g}$ for $30 \mathrm{~min}$ at $4{ }^{\circ} \mathrm{C}$. The resulting supernatant was centrifuged at $100,000 \mathrm{~g}$ for $1 \mathrm{~h}$ at $4{ }^{\circ} \mathrm{C}$. The pellet was resuspended in $20 \mathrm{mM}$ Tris- $\mathrm{HCl} \mathrm{pH} 7.4$ containing $100 \mathrm{mM} \mathrm{NaCl}$ at $250 \mu \mathrm{l} / \mathrm{g}$ tissue and centrifuged at $100,000 \mathrm{~g}$ for $30 \mathrm{~min}$ at $4{ }^{\circ} \mathrm{C}$. The final pellet was resuspended in buffer at $15 \mu \mathrm{l} / \mathrm{g}$ tissue for cryo-EM and $150 \mu \mathrm{l} / \mathrm{g}$ tissue for immuno-EM.

\section{Electron cryo-microscopy}

Extracted tau filaments were applied to glow-discharged holey carbon grids (Quantifoil Au R1.2/1.3, 300 mesh) and 
plunge-frozen in liquid ethane using an FEI Vitrobot Mark IV. Images were acquired on a Gatan K2-Summit detector in counting mode using an FEI Titan Krios at $300 \mathrm{kV}$. A GIFquantum energy filter (Gatan) was used with a slit width of $20 \mathrm{eV}$ to remove inelastically scattered electrons. Further details are given in Online Resource 2.

\section{Helical reconstruction}

Movie frames were corrected for gain reference, motioncorrected, and dose-weighted using MOTIONCOR2 [44]. Aligned, non-dose-weighted micrographs were used to estimate the contrast transfer function in Gctf [43]. All subsequent image-processing steps were performed using helical reconstruction methods in RELION 2.1 [24, 34]. Filaments were picked manually. For case 2, PHFs and SFs were picked as separate data sets. For cases 3 and 16, filaments were collected as a single data set; PHFs and SFs were subsequently separated by reference-free $2 \mathrm{D}$ classification of segments comprising an entire helical crossover. PHF and SF segments were re-extracted using box sizes of 200 or 270 pixels and an inter-box distance of approximately 14 $\AA$ A. Reference-free 2D classification was performed and segments contributing to suboptimal 2D class averages were discarded. For cases 2 and 16, the initial 3D models were constructed de novo from 2D class averages of segments comprising entire helical crossovers, and low-pass filtered to $40 \AA$. For case 3, the PHF and SF reconstructions from case 1 (PDB accession numbers 503L and 503T) [14] were lowpass filtered to $10 \AA$ and used as the initial 3D models. 3D classification with local optimisation of helical twist and rise was performed to remove segments contributing to suboptimal 3D class averages, and the selected segments were used for 3D auto-refinement with optimisation of the helical twist. A value of $10 \%$ was used for the helical z percentage parameter. For case 16, particle polishing was performed, followed by further $3 \mathrm{D}$ auto-refinement. The final reconstructions were sharpened using the standard post-processing procedures in RELION and helical symmetry was imposed using the RELION helix toolbox [24]. Finally, overall resolution estimates were calculated from Fourier shell correlations at 0.143 between two independently refined half-maps, using phase randomization to correct for the convolution effects of a generous, soft-edged solvent mask [5]. Local resolution estimates were obtained using the same-phase randomization procedure, but with a soft spherical mask that was moved over the entire map. For further details, see Online Resource 2. Cryo-EM maps for case 2 have been deposited in the Electron Microscopy Data Bank (EMDB) under accession numbers EMD-0259 for PHF and EMD-0260 for SF.

\section{Model building and refinement}

Compared to [14], the PHF and SF reconstructions from the frontal cortex of AD patient 2 showed clear densities corresponding to two additional residues at the $\mathrm{N}$-terminus (G273 and K274 from 3R tau; or G304 and S305 from 4R tau) and two residues at the C-terminus (R379 and E380) of the protofilament core. These residues were added to the PHF and SF models from the frontal cortex of AD patient 1 [14] (PDB accession numbers 503L and 503T) using COOT [11]. The new models were then refined against the PHF and SF reconstructions from patient 2 using targeted realspace refinement in COOT. The models were subsequently translated to give stacks of three consecutive monomers to preserve nearest-neighbour interactions for the middle chains in subsequent Fourier space refinements in REFMAC [30]. Local symmetry restraints were imposed to keep all $\beta$-strand rungs identical. Side-chain clashes were detected using MOLPROBITY [6] and corrected by iterative cycles of real-space refinements in COOT and Fourier space refinements in REFMAC. Separate model refinements were performed against single half-maps, and the resulting models compared with the other half-maps to confirm the absence of overfitting. The final models were stable in refinements without additional restraints. Further details are given in Online Resource 3. Refined atomic models for case 2 have been deposited in the Protein Data Bank (PDB) under accession numbers $6 \mathrm{HRE}$ for PHF and 6HRF for SF.

\section{Immuno-gold negative-stain electron microscopy}

Extracted tau filaments were deposited on glow-discharged 400 mesh formvar/carbon film-coated copper grids (EM Sciences CF400-Cu) for $40 \mathrm{~s}$, blocked for $10 \mathrm{~min}$ with PBS $+0.1 \%$ gelatin, and incubated with primary antibody (1:50) in blocking buffer, essentially as described [20]. Primary antibodies were BR136 (raised against residues 244-257) [12], Anti-4R (raised against residues 275-291, with D279) [10], BR135 (raised against residues 323-335) [21], and TauC4 (raised against residues 354-369) [38]. Where stated, grids were incubated for $5 \mathrm{~min}$ with $0.4 \mathrm{mg} /$ $\mathrm{ml}$ pronase (Sigma) in PBS at room temperature and washed with blocking buffer, prior to blocking. Following incubation with primary antibodies, grids were washed with blocking buffer and incubated with $10 \mathrm{~nm}$ gold-conjugated anti-rabbit IgG (Sigma) diluted 1:20 in blocking buffer. The grids were then washed with water and stained with $2 \%$ uranyl acetate for $40 \mathrm{~s}$. Images were acquired at $11,000 \times$ and $15,000 \times$, with a defocus value of $-1.4 \mu \mathrm{m}$ with Gatan Orius SC200B or Gatan Ultrascan 1,000 CP CCD detectors using a Tecnai G2 Spirit at $120 \mathrm{kV}$. To distinguish non-specific background levels of isolated gold beads from specific 
labelling of the filaments, we only considered filaments with more than six gold beads to be positively labelled.

\section{Whole-exome sequencing}

DNAs of the AD cases were sequenced at the Center for Medical Genomics of Indiana University School of Medicine. Target enrichment made use of the SureSelectXT human all exon library (V6, $58 \mathrm{Mb}$, Agilent) and highthroughput sequencing was carried out using a HiSeq4000 $(2 \times 75$ bp paired-end configuration, Illumina). Bioinformatics analyses were performed as described [13]. There were no pathogenic mutations in $M A P T$. Table 1 summarises the findings for APP, PSEN1, PSEN2, and APOE.
Table 1 Cases of Alzheimer's disease

\begin{tabular}{|c|c|c|c|c|c|c|c|}
\hline Case & Gender & $\begin{array}{c}\text { Age at } \\
\text { death (y) }\end{array}$ & $\begin{array}{c}\text { PMI } \\
\text { (h) }\end{array}$ & $\boldsymbol{A P P}$ & PSEN1 & PSEN2 & $\begin{array}{c}A P O E \\
\text { haplotypes }\end{array}$ \\
\hline 1 & $\mathrm{~F}$ & 74 & 5.3 & wt & wt & wt & $\varepsilon 3 / \varepsilon 4$ \\
\hline 2 & $\mathrm{~F}$ & 82 & 4.5 & wt & wt & wt & $\varepsilon 4 / \varepsilon 4$ \\
\hline 3 & M & 79 & 3.0 & wt & wt & wt & $\varepsilon 3 / \varepsilon 4$ \\
\hline 4 & M & 84 & 2.6 & wt & wt & wt & $\varepsilon 3 / \varepsilon 4$ \\
\hline 5 & $\mathrm{~F}$ & 87 & 7.7 & wt & wt & wt & $\varepsilon 3 / \varepsilon 3$ \\
\hline 6 & M & 71 & 2.0 & wt & wt & wt & $\varepsilon 3 / \varepsilon 4$ \\
\hline 7 & $\mathrm{~F}$ & 84 & 32.8 & wt & wt & wt & $\varepsilon 3 / \varepsilon 4$ \\
\hline 8 & $\mathrm{~F}$ & 93 & 18.5 & wt & wt & wt & $\varepsilon 3 / \varepsilon 3$ \\
\hline 9 & $\mathrm{~F}$ & 77 & 2.5 & wt & wt & wt & $\varepsilon 3 / \varepsilon 3$ \\
\hline 10 & $\mathrm{~F}$ & 90 & 8.5 & wt & wt & wt & $\varepsilon 3 / \varepsilon 3$ \\
\hline 11 & $\mathrm{~F}$ & 79 & 3.0 & wt & wt & wt & $\varepsilon 4 / \varepsilon 4$ \\
\hline 12 & $\mathrm{~F}$ & 86 & 22.0 & wt & wt & wt & $\varepsilon 3 / \varepsilon 4$ \\
\hline 13 & $\mathrm{~F}$ & 74 & 2.8 & wt & wt & wt & $\varepsilon 4 / \varepsilon 4$ \\
\hline 14 & $\mathrm{~F}$ & 57 & 5.5 & wt & wt & wt & $\varepsilon 3 / \varepsilon 3$ \\
\hline 15 & $\mathrm{~F}$ & 86 & 12.3 & wt & wt & wt & $\varepsilon 4 / \varepsilon 4$ \\
\hline 16 & $\mathrm{~F}$ & 54 & 5.3 & V717F & wt & wt & $\varepsilon 3 / \varepsilon 3$ \\
\hline 17 & M & 46 & 4.0 & V717F & wt & wt & $\varepsilon 3 / \varepsilon 3$ \\
\hline 18 & $\mathrm{M}$ & 63 & 2.1 & wt & wt & wt & $\varepsilon 4 / \varepsilon 4$ \\
\hline 19 & $\mathrm{M}$ & 65 & 3.4 & wt & wt & wt & $\varepsilon 3 / \varepsilon 4$ \\
\hline
\end{tabular}

Wild-type (wt), no known pathogenic mutations were detected. Cases 1, 2, 3, and 16 were used for cryo-EM (highlighted in yellow)

$P M I$ post-mortem interval
PHFs

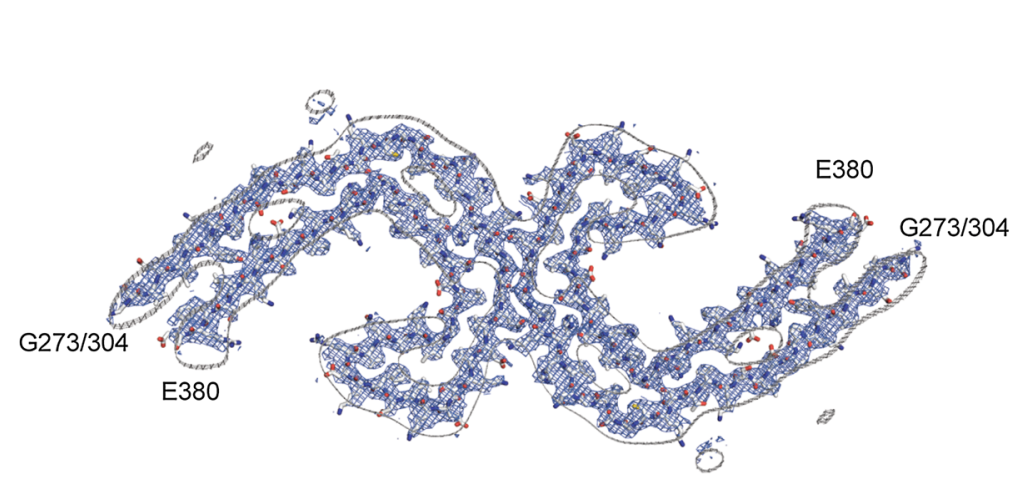

SFs

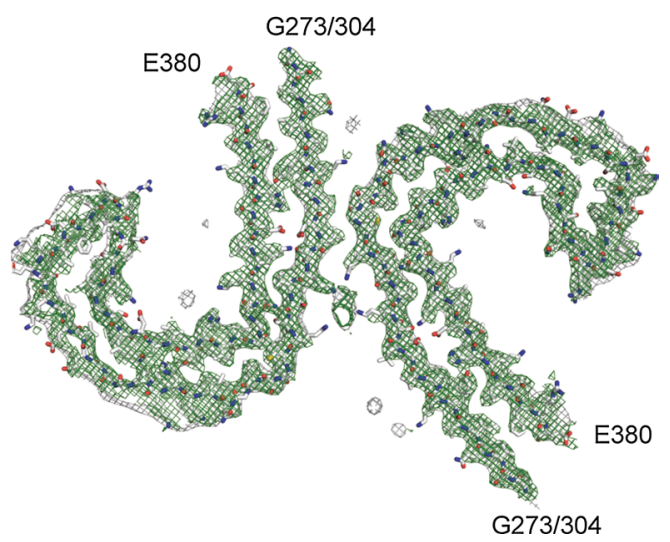

Fig. 1 Cryo-EM densities and atomic models of PHFs and SFs from the frontal cortex of AD case 2. PHFs and SFs from case 2 are resolved to $3.2 \AA$ and $3.3 \AA$, respectively. Sharpened, high-resolution maps are shown in blue (PHF) and green (SF). Unsharpened $4.5 \AA$ low-pass filtered densities are shown in grey. The models comprise G273-E380 of 3R tau and G304-E380 of 4R tau 


\section{Results}

\section{Electron cryo-microscopy}

We first used cryo-EM to image tau filaments extracted from the frontal cortex of an individual with sporadic AD (Fig. 1, case 2 in Table 1). PHFs and SFs were present in a ratio of approximately 4:1. Using helical reconstruction in RELION [24], we determined the structures of PHFs to $3.2 \AA$ and of SFs to $3.3 \AA$ resolution (Fig. 1, Online Resources 1, 2 and 3). As reported before [14] for filaments extracted from a case of sporadic AD (case 1 in Table 1), the core structure comprised V306-F378 of tau in a combined cross- $\beta / \beta$-helix, $\mathrm{C}$-shaped fold (Online Resource 4). Moreover, the higher resolution structures of tau filaments reported here showed additional densities corresponding to G304 and S305 from R2 (or G273 and K274 from R1), as well as R379 and E380 from the sequence after R4, which were not as well resolved in the structures from AD case 1 [14]. The first $\beta$-strand of the protofilament structure begins at K274 of R1 or at S305 of R2 and ends at K311, whereas the eighth $\beta$-strand extends from N368-E380. Weaker densities extending from the $\mathrm{N}$ - and C-terminal regions of the core, described for filaments extracted from AD case 1 [14], were also observed, as were the densities interacting with the sidechains of K317, T319, and K321. In PHFs, the side chains of K331 from one protofilament projected between the side chains of Q336 and E338 of the other protofilament, suggesting additional interactions that stabilize the protofilament interface. Moreover, in the protofilament interface of the PHF, the higher resolution structure revealed the presence of extra densities extending from the side chains of K331 on one protofilament to the backbone atoms of V337 of the other protofilament (Online Resource 5). This density may correspond to a water molecule or a post-translational modification of K331, such as mono-methylation.

We also used cryo-EM to determine the structures of tau filaments extracted from the frontal cortex of a third individual with sporadic AD (case 3 in Table 1) and an individual with dominantly inherited $\mathrm{AD}$ (mutation V717F in $A P P$; case 16 in Table 1). In both individuals, PHFs and SFs were present in a ratio of approximately 4:1. Despite lower resolution, the structures of PHFs and SFs were the same as those of tau filaments from cases 1 and 2 (Fig. 2, Online Resource 2). A side-by-side comparison of PHF and SF reconstructions from cases 1-4 showed the presence of extra densities bordering the solvent-exposed side chains of R349 and K375, and of H362 and K369 within each C-shape (Fig. 2, Online Resource 2).

\section{Immuno-gold negative-stain electron microscopy}

To extend our analysis to a larger number of $\mathrm{AD}$ cases and additional brain regions from the first case studied by
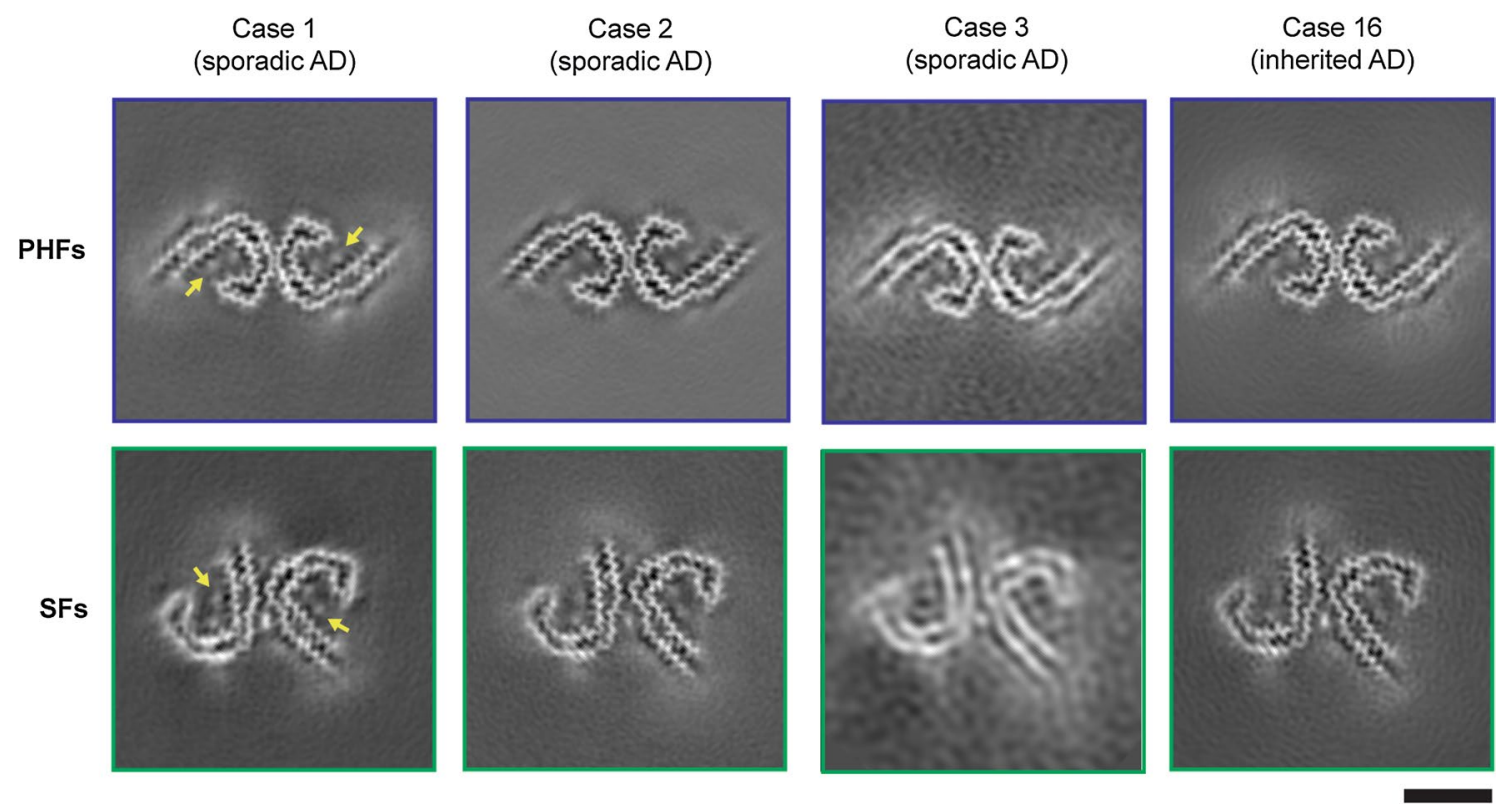

Fig. 2 Cryo-EM structures of PHFs and SFs from the frontal cortex of $\mathrm{AD}$ cases 1,2,3, and 16. All structures show identical pairs of $\mathrm{C}$-shaped protofilaments and the same inter-protofilament packing in PHFs and SFs. Cases 1, 2, and 3 had sporadic AD, whereas case 16 had inherited AD (mutation V717F in $A P P$ ). The filament structures of case 1 are from (14); the structures from cases 2, 3, and 16 are first described here. All cases had a majority of PHFs and a minority of SFs. Yellow arrows indicate the extra densities, which are present in PHFs and SFs from all four cases, bordering the solvent-exposed side chains of R349 and K375, and of H362 and K369. Scale bar, $50 \AA$ 
a

\begin{tabular}{|l|l|l|l|l|l|l|l|l|}
\hline 1 & 45 & 74 & 103 & \multicolumn{3}{c}{244} & \multicolumn{3}{c|}{369} \\
\hline & N1 & N2 & & R1 & R2 & R3 & R4 & \\
\hline
\end{tabular}

b
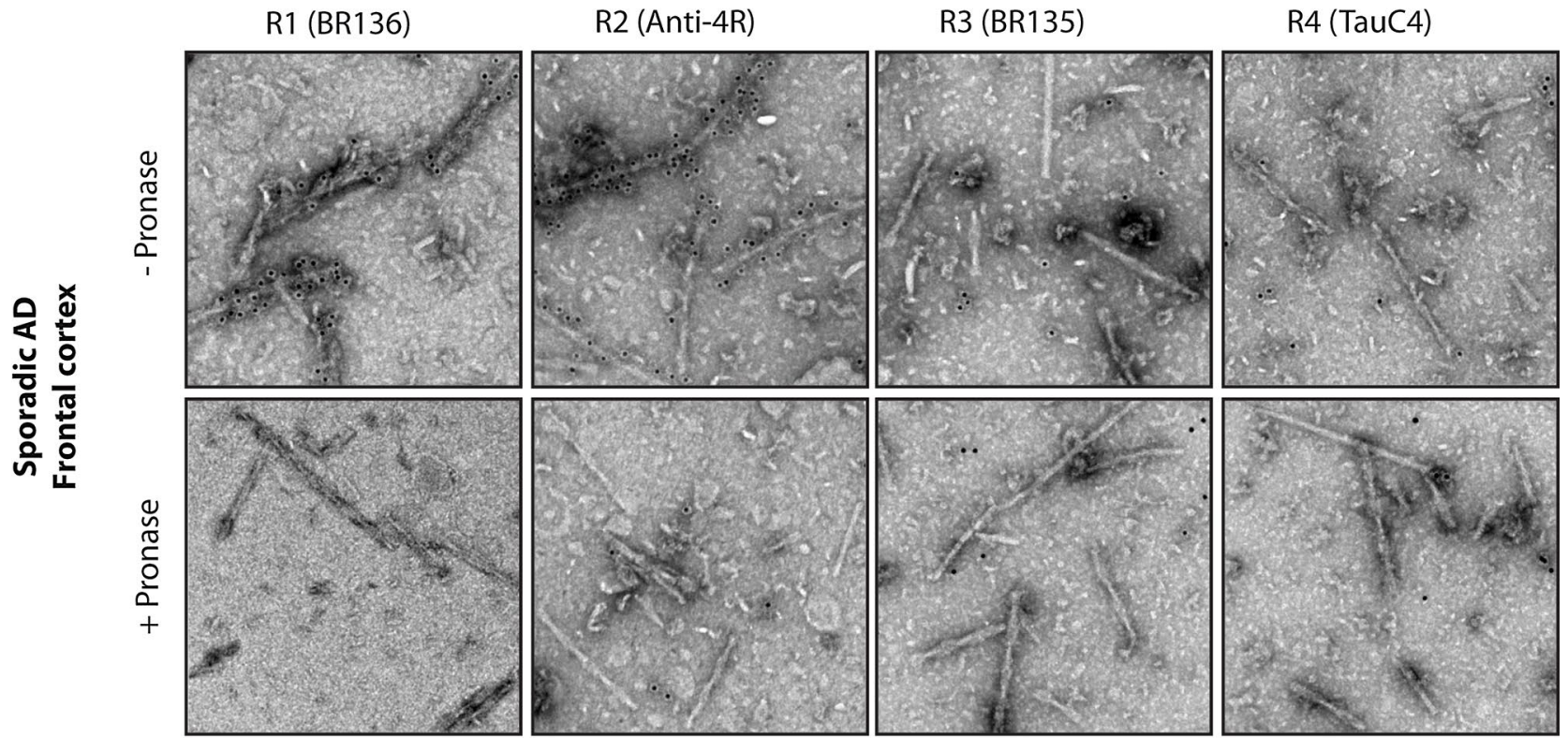

C
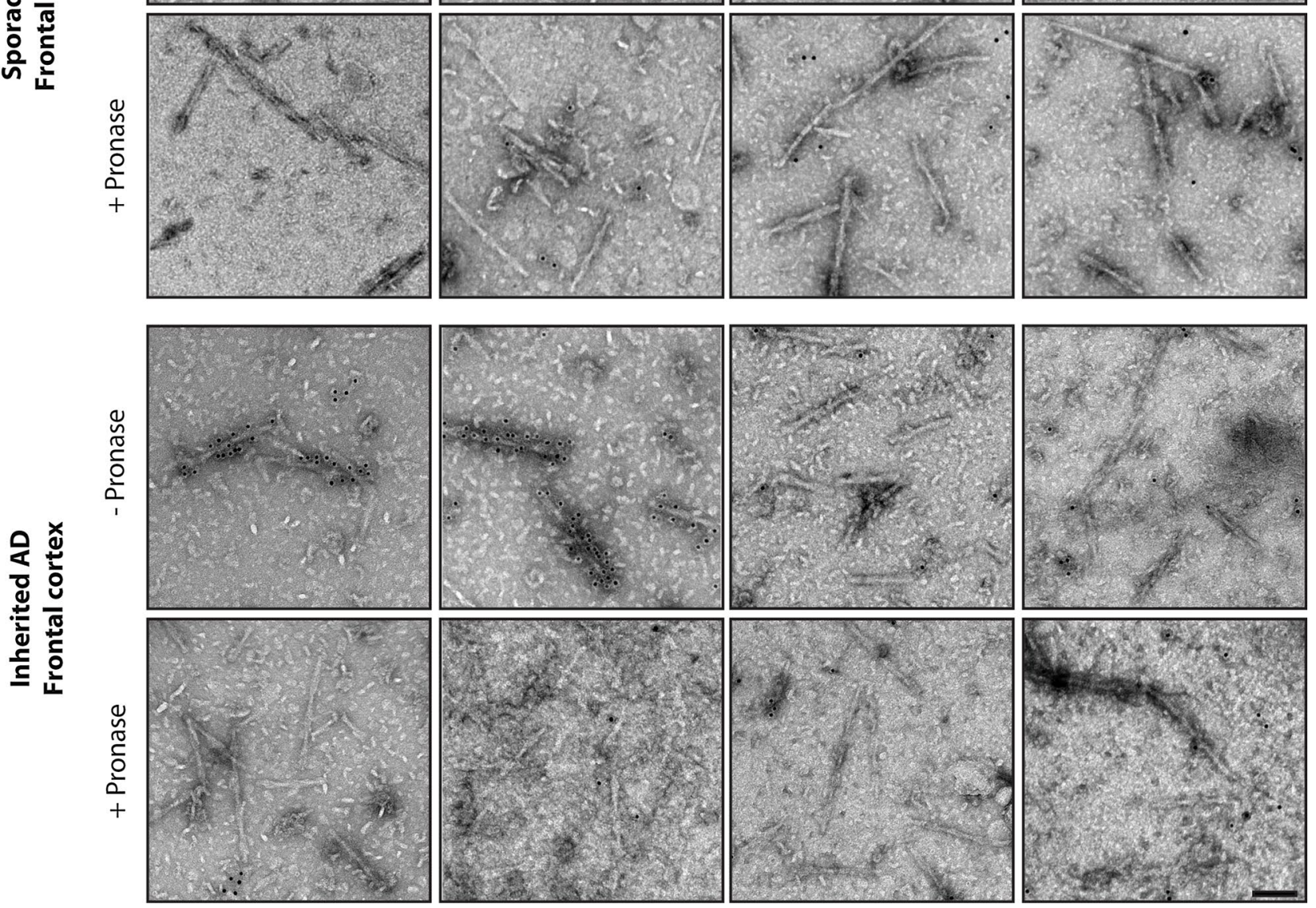

Fig. 3 Immuno-EM of tau filaments from the frontal cortex of sporadic and inherited AD cases. a 441 amino acid isoform of human tau and epitopes of repeat-specific antibodies. The amino-terminal inserts are labelled N1 and N2, with the microtubule-binding repeats being R1-R4. Black lines indicate the epitopes of antibodies BR136 (R1), Anti-4R (R2), BR135 (R3) and TauC4 (R4) that were used for immuno-EM. b, c Representative images from sporadic (b) and

cryo-EM [14], we used immuno-EM to assess filament morphologies and the labelling of extracted filaments by repeat-specific anti-tau antibodies. We used antibodies specific for residues 244-257 of R1 (BR136), residues inherited (c) cases of AD before (-) and after (+) pronase treatment. Frontal cortex from 15 cases of typical sporadic AD and two cases of inherited $\mathrm{AD}$ (mutation $\mathrm{V} 717 \mathrm{~F}$ in $A P P$ ) were studied. Tau filaments from all the cases were decorated by BR 136 and Anti-4R before pronase treatment, but not by BR135 or TauC4. Pronase treatment abolished labelling by BR136 and Anti-4R. PHFs were in the majority and SFs in the minority. Scale bar, $100 \mathrm{~nm}$

275-291, with D279, of R2 (Anti-4R), residues 323-335 of R3 (BR135), and residues 354-369 of R4 (TauC4) of tau, all of which label tau bands on Western blots of 
dispersed filaments [12,14] (Fig. 3a, Online Resource 6). However, linear epitopes buried in the cores of tau filaments are not accessible to antibodies, in contrast to epitopes located in the fuzzy coat, which give positive labelling [14]. Moreover, pronase treatment removes the fuzzy coat, abolishing this positive labelling.

Besides tau filaments from the four cases analysed by cryo-EM (cases 1-3 and 16), we also looked at tau filaments from the frontal cortex of 12 additional cases of sporadic $\mathrm{AD}$ (patients 4-15) and one more case of dominantly inherited $\mathrm{AD}$ (V717F in APP, patient 17) (Table 1). In all cases, extracted filaments consisted of a majority of PHFs and a minority of SFs. By negative-stain EM, antibodies BR136 and Anti-4R decorated the filaments from all cases before, but not after, pronase treatment. By contrast, BR135 and TauC4 did not decorate PHFs or SFs from the frontal cortex of cases 4-15 or case 17, either before or after pronase treatment (Fig. 3b, c, Online Resource 7). This suggests that residues 323-335 of R3 and 354-369 of R4 of tau are in the filament core, whereas residues 244-257 of R1 and 275-291 of R2 of tau lie in the fuzzy coat. These findings are consistent with the presence of the same tau sequences in the core of tau filaments in the frontal cortex of all the analysed cases (17 cases of sporadic AD and 2 cases of dominantly inherited AD).

To extend our findings to different brain regions, we studied tau filaments from temporal, occipital, parietal, and cingulate cortices, as well as from thalamus, substantia innominata, and putamen of case 1 of sporadic $\mathrm{AD}$, whose frontal cortex was used for cryo-EM and immuno-EM (Figs. 2, 4, Online Resource 7). In all regions, a majority of PHFs and a minority of SFs were in evidence. By immunoEM, filaments were labelled by BR 136 and Anti-4R before, but not after, pronase treatment, and not by BR135 or TauC4. This indicates that, in all brain regions examined, residues 323-335 of R3 and 354-369 of R4 are present in the core of the filaments, as in all cases of AD examined.

PCA is an atypical form of sporadic AD with abundant tau filaments in the occipital cortex [9]. As in typical AD, a majority of PHFs and a minority of SFs were present in frontal and occipital cortices of cases 18 and 19. Tau filaments were decorated by BR136 and Anti-4R, but not by BR135 or TauC4 (Fig. 5, Online Resource 7). Following pronase treatment, the decoration by BR136 and Anti-4R was lost, suggesting also, for these cases, the presence of residues 323-335 of R3 and 354-369 of R4 of tau in the filament core.

\section{Discussion}

The cryo-EM structures of the core of PHFs and SFs from the frontal cortex of four typical cases of $\mathrm{AD}$ (three sporadic and one inherited) showed a common protofilament fold. The filament structures from case 1 were reported in [14]. The structures from case 2 were of higher resolution than
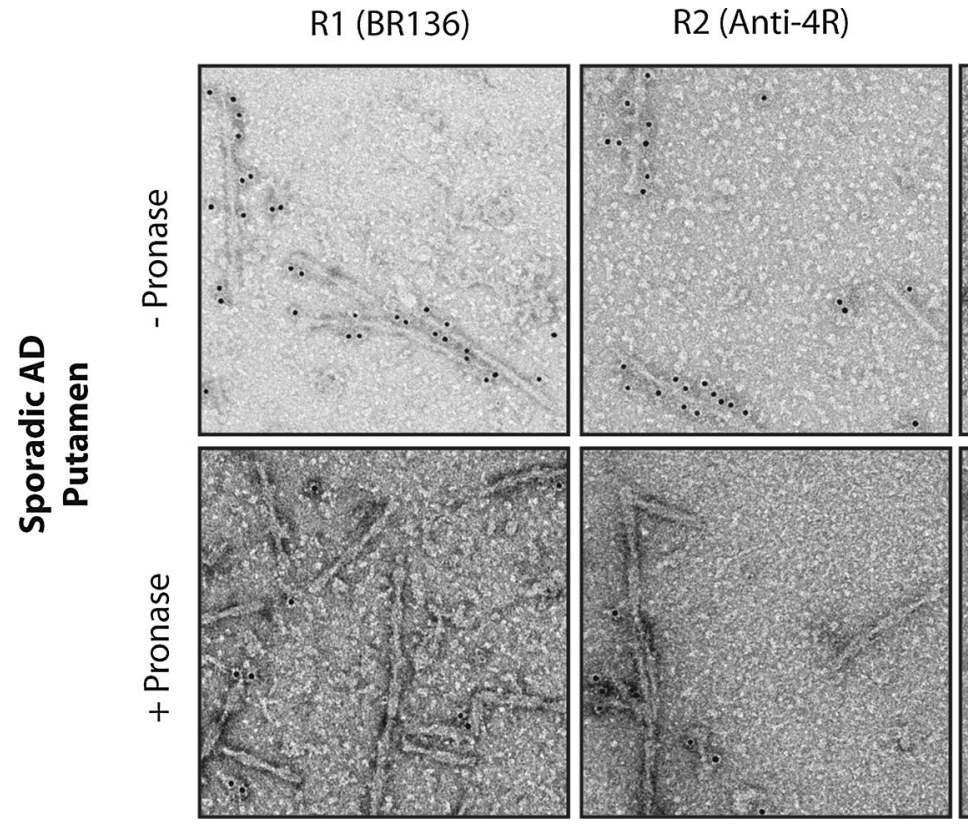

R3 (BR135)
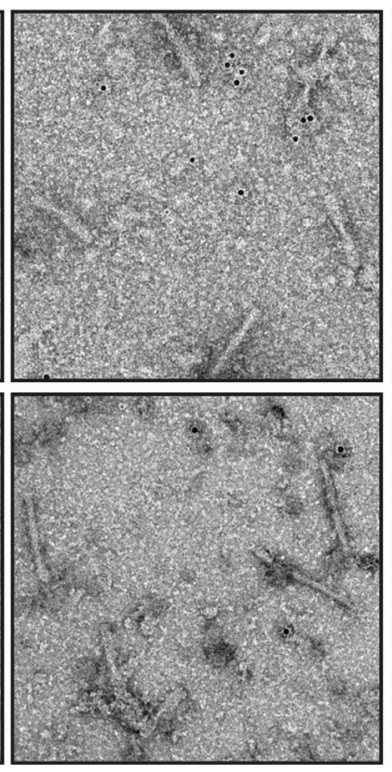

R4 (TauC4)

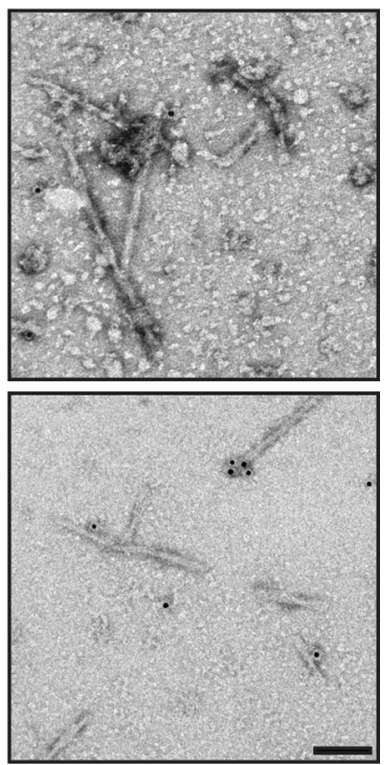

Fig. 4 Immuno-EM of tau filaments from the putamen of AD case 1. Representative images before $(-)$ and after $(+)$ pronase treatment. In addition to frontal cortex and putamen, temporal cortex, parietal cortex, cingulate cortex, thalamus, and substantia innominata from case 1 were also analysed. Tau filaments from all brain regions were decorated by BR136 and Anti-4R before pronase treatment, but not by BR135 or TauC4. Pronase treatment abolished labelling by BR136 and Anti-4R. PHFs were in the majority and SFs in the minority. Scale bar, $100 \mathrm{~nm}$ 


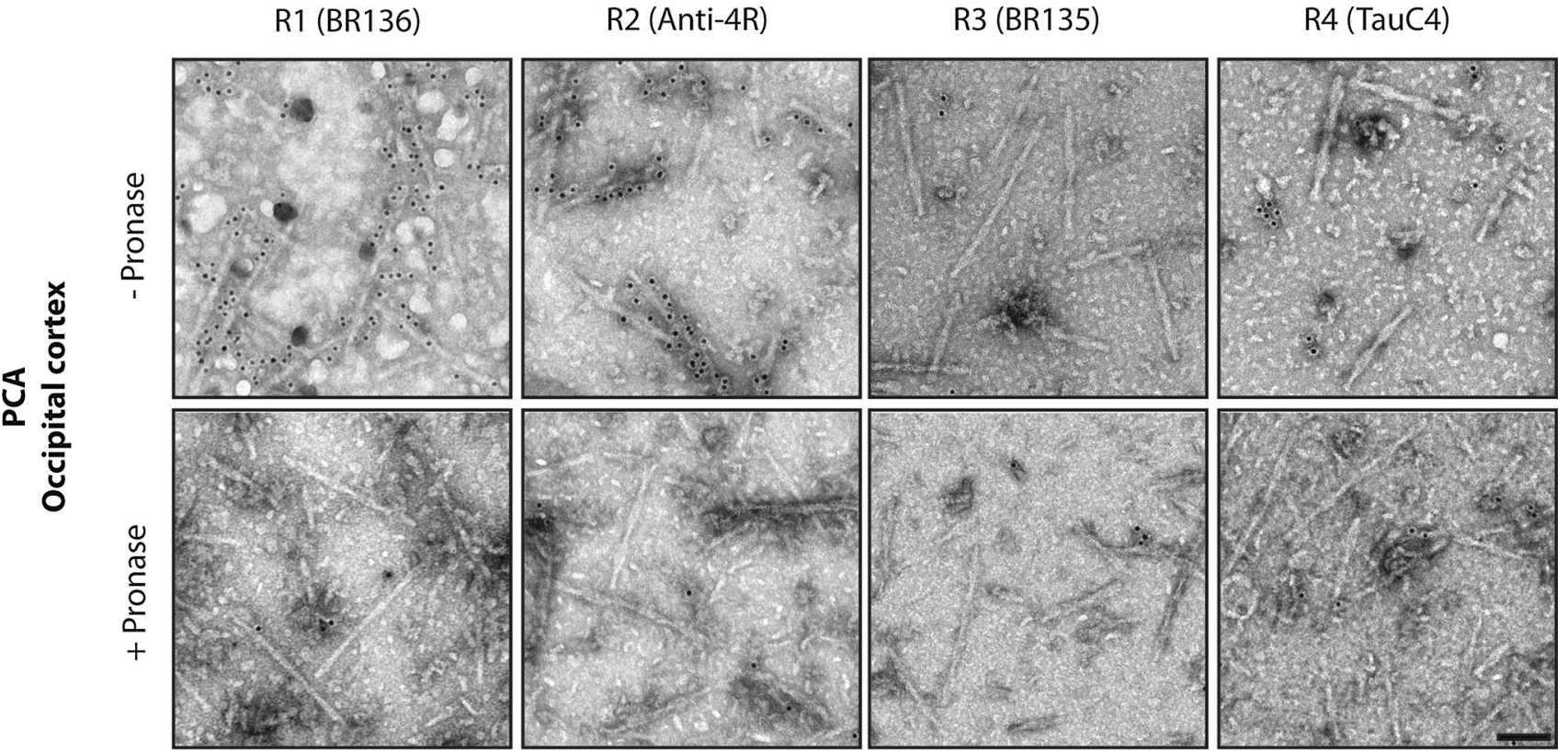

Fig. 5 Immuno-EM of tau filaments from the occipital cortex of PCA cases. Representative images before $(-)$ and after $(+)$ pronase treatment. Occipital cortex from two cases of PCA, an atypical form of sporadic AD, was studied. Tau filaments from both cases were dec-

those of case 1, but the resolutions of structures from cases 3 and 16 were lower. The achievable resolution of filament structures from a given $\mathrm{AD}$ case is mainly determined by the number of filaments that can be extracted from brain. PHFs and SFs from all four cases were made of two protofilaments with a common cross- $\beta / \beta$-helix $C$-shaped architecture. The interfaces between the two protofilaments of both PHFs and SFs were the same in all four cases. These findings show that the PHF and SF structures are identical between sporadic (irrespective of $A P O E$ genotype) and inherited cases of $\mathrm{AD}$.

The higher resolution cryo-EM structures of PHFs and SFs from case 2 showed the presence of two additional residues at the N-terminus, and two additional residues at the C-terminus of the ordered core of the protofilament. At the C-terminus, these residues were R379 and E380 from the sequence after R4. Because AD filaments contain a mixture of all six brain tau isoforms [20], the additional density at the $\mathrm{N}$-terminus arose from a mixture of $3 \mathrm{R}$ and $4 \mathrm{R}$ tau isoforms. It comprised G304 and S305 from R2 in 4R tau, and G273 and K274 from R1 in 3R tau. The additional cryo-EM density at the $\mathrm{N}$-terminus was consistent with the presence of a glycine and a mixture of a partially disordered serine and a lysine. Weaker densities extended from the $\mathrm{N}$ - and $\mathrm{C}$-terminal regions of the core, which could accommodate additional tau residues in more dynamic or transiently occupied structures, as reported in the structures from $\mathrm{AD}$ case 1 [14]. Densities interacting with the sidechains of K317, T319, and K321 were also observed, which we previously orated by BR136 and Anti-4R before pronase treatment, but not by BR135 or TauC4. Pronase treatment abolished labelling by BR136 and Anti-4R. PHFs were in the majority and SFs in the minority. Scale bar, $100 \mathrm{~nm}$

suggested could be residues ${ }^{7} \mathrm{EFE}^{9}$ of tau, giving rise to the discontinuous MC1 epitope [14]. Similar densities were also found in the structures of tau filaments from PiD [12].

Since cryo-EM is a low-throughput technique, we also used immuno-EM to study tau filaments from the frontal cortex of the four cases used for cryo-EM, plus another 15 cases of AD. They represented both typical and atypical cases, and a variety of $A P O E$ genotypes. It is important to combine both techniques, because immuno-EM does not provide high-resolution structural information. In all cases, PHFs were in the majority and SFs in the minority. They were decorated by BR136 and Anti-4R, but not by BR135 or TauC4, indicating that R3 and R4 form part of the filament core, whereas the $\mathrm{N}$-terminal regions of $\mathrm{R} 1$ and $\mathrm{R} 2$ lie within the fuzzy coat. Importantly, the immuno-EM labelling of tau filaments from the cases used for cryo-EM was indistinguishable from that of the other cases studied, including PCA, an atypical form of sporadic AD. Moreover, for case 1, brain regions other than frontal cortex, including subcortical areas, gave an identical decoration pattern and contained a majority of PHFs. Thus, the core of all tau filaments contained R3 and R4.

The cryo-EM structures of tau filaments from individuals with end-stage $\mathrm{AD}$ do not tell us how the assembly process may have started. Since tau is hydrophilic [40], it is not surprising that the formation of filaments by recombinant protein requires cofactors, such as heparin [19, 31]. Negatively charged cofactors and/or chemical modifications of tau may 
be needed for the ordered assembly of tau into filaments in human brain. The extra densities bordering the positively charged side chains of solvent-exposed amino acids in the cryo-EM maps of PHFs and SFs from all four cases may be such factors. Once a seed has formed, tau may be able to assemble and propagate [23, 28], which may also require such cofactors and/or chemical modifications. In a mouse model transgenic for human mutant P301S tau, short tau filaments had the greatest seeding activity in non-neuronal cells expressing full-length human P301S tau [25]. It remains to be seen if this is also the case for AD.

The present findings suggest that the filament cores from multiple brain regions of sporadic and inherited cases of $\mathrm{AD}$, as well as of atypical sporadic cases, contain identical tau sequences. They are consistent with the existence of a common fold for tau filaments in AD. Tau is an intracellular protein. Its accumulation may involve mechanisms that differ from those that facilitate the seeding of proteins that form amyloids extracellularly. For $\mathrm{PrP}^{\mathrm{Sc}}$ and $\mathrm{A} \beta$, structurally heterogeneous assemblies, also referred to as conformational clouds, have been described [7, 32, 39]. Knowledge of the structural differences between assemblies of $\operatorname{PrP}^{\mathrm{Sc}}$ and $\mathrm{A} \beta$ must await the determination of their high-resolution structures from brain.

Distinct tau filament folds are found in specific diseases. The cryo-EM structures of tau filaments from the brain of an individual with PiD revealed that they adopt a fold that is different from the AD fold [12]. Immuno-EM results for eight additional cases of PiD revealed a common ordered filament core comprising most of R1, as well as the whole of R3 and R4. Therefore, specific molecular conformers of aggregated tau may define different diseases, without there being a significant variation in filament structures between individuals with a given disease. This understanding will inform the design of diagnostic ligands of higher specificity and the development of therapies targeting tau assembly. It remains to be seen if what is true of $\mathrm{AD}$ and $\mathrm{PiD}$ also applies to other human tauopathies.

Acknowledgements We thank the patients' families for donating brain tissues; M.R. Farlow for clinical evaluation; F. Epperson, R.M. Richardson, and U. Kuederli for brain collection and analysis; A.W.P. Fitzpatrick for cryo-EM data collection of AD cases 2 and 3; M. Hasegawa for antibody TauC4; S. Chen, C. Savva, G. Cannone, and K. Dent for support with electron microscopy; T. Darling and J. Grimmett for help with computing; R.A. Crowther for helpful discussions. M.G. is an Honorary Professor in the Department of Clinical Neurosciences of the University of Cambridge. This work was supported by the UK Medical Research Council (MC_UP_A025_1013 to S.H.W.S. and MC_U105184291 to M.G.), the European Union (Joint Programme-Neurodegeneration Research REfrAME to B.F. and M.G. and the Innovative Medicines Initiative 2 IMPRiND, project 115881, to M.G.), Eli Lilly and Company (to M.S. and M.G.), the US National Institutes of Health (Grant P30-AG010133 to B.G.), the Department of Pathology and Laboratory Medicine, Indiana University School of Medicine (to B.G.), and an Alzheimer's Association Zenith Award (to R.V.). We acknowledge DIAMOND for access to and support of the cryo-EM facilities at the UK electron Bio-Imaging Centre (eBIC), proposal EM17434, funded by the Wellcome Trust, the MRC, and the BBSRC.

Author contributions B.G. performed neuropathology; H.J.G. and R.V. carried out genetic analysis; B.F. and M.S. extracted tau filaments; B.F. and W.Z. performed cryo-EM; B.F., W.Z., A.G.M., and S.H.W.S. analysed cryo-EM data; B.F. refined atomic models; B.F., W.Z., and M.S. conducted immuno-EM; S.H.W.S. and M.G. supervised the project.

Open Access This article is distributed under the terms of the Creative Commons Attribution 4.0 International License (http://creativeco mmons.org/licenses/by/4.0/), which permits unrestricted use, distribution, and reproduction in any medium, provided you give appropriate credit to the original author(s) and the source, provide a link to the Creative Commons license, and indicate if changes were made.

\section{References}

1. Benson DF, Davis RJ, Snyder BD (1988) Posterior cortical atrophy. Arch Neurol 45:789-793

2. Berriman J, Serpell LC, Oberg KA, Fink AL, Goedert M, Crowther RA (2003) Tau filaments from human brain and from in vitro assembly of recombinant protein show cross-beta structure. Proc Natl Acad Sci (USA) 100:9034-9038

3. Braak H, Braak E (1991) Neuropathological stageing of Alzheimer-related changes. Acta Neuropathol 82:239-259

4. Brion J-P, Passareiro H, Nunez J, Flament-Durand J (1985) Mise en évidence immunologique de la protéine tau au niveau des lésions de dégénérescence neurofibrillaire de la maladie d'Alzheimer. Arch Biol (Bruxelles) 95:229-235

5. Chen S, McMullan G, Faruqi AR, Murshudov GN, Short JM, Scheres SH, Henderson R (2013) High-resolution noise substitution to measure overfitting and validate resolution in 3D structure determination by single particle electron cryomicroscopy. Ultramicroscopy 135:24-35

6. Chen VB, Arendall WB 3rd, Headd JJ, Keedy DA, Immormino RM, Kapral GJ, Murray LW, Richardson JS, Richardson DC (2010) MolProbity: all-atom structure validation for macromolecular crystallography. Acta Crystallogr D 66:12-21

7. Collinge $\mathbf{J}$ (2016) Mammalian prions and their wider relevance in neurodegenerative diseases. Nature 539:217-226

8. Crowther RA (1991) Straight and paired helical filaments in Alzheimer disease have a common structural unit. Proc Natl Acad Sci (USA) 88:2288-2292

9. Crutch SJ, Lehmann M, Schott JM, Rabinovici GD, Rossor MN, Fox NC (2012) Posterior cortical atrophy. Lancet Neurol 11:170-178

10. Dan A, Takahashi M, Masuda-Suzukake M, Kametani F, Nonaka T, Kondo H, Akiyama H, Arai T, Mann DM, Saito Y et al (2013) Extensive deamidation at asparagine residue 279 accounts for weak immunoreactivity of tau with RD4 antibody in Alzheimer's disease brain. Acta Neuropathol Commun 1:54

11. Emsley P, Lohkamp B, Scott WG, Cowtan K (2010) Features and development of Coot. Acta Crystallogr D 66:486-501

12. Falcon B, Zhang W, Murzin AG, Murshudov G, Garringer HJ, Vidal R, Crowther RA, Ghetti B, Scheres SHW, Goedert M (2018) Structures of filaments from Pick's disease reveal a novel tau protein fold. Nature 561:137-140 
13. Farlow JL, Robak LA, Hetrick K, Bowling K, Boerwinkle E, Coban-Akdemir ZH, Gambin T, Gibbs RA, Gu S, Jain P et al (2016) Whole-exome sequencing in familial Parkinson disease. JAMA Neurol 73:68-75

14. Fitzpatrick AWP, Falcon B, He S, Murzin AG, Murshudov G, Garringer HJ, Crowther RA, Ghetti B, Goedert M, Scheres SHW (2017) Cryo-EM structures of tau filaments from Alzheimer's disease. Nature 547:185-190

15. Galton CJ, Patterson K, Xuereb JH, Hodges JR (2000) Atypical and typical presentations of Alzheimer's disease: a clinical, neuropsychological, neuroimaging and pathological study of 13 cases. Brain 123:484-498

16. Glenner GG, Wong CW (1984) Alzheimer's disease: initial report of the purification and characterization of a novel cerebrovascular amyloid protein. Biochem Biophys Res Commun 120:885-890

17. Goate A, Chartier-Harlin MC, Mullan M, Brown J, Crawford F, Fidani L, Giuffra L, Haynes A, Irving N, James L et al (1991) Segregation of a missense mutation in the amyloid precursor protein gene with familial Alzheimer's disease. Nature 349:704-706

18. Goedert M, Eisenberg DS, Crowther RA (2017) Propagation of tau aggregates and neurodegeneration. Annu Rev Neurosci 40:189-210

19. Goedert M, Jakes R, Spillantini MG, Hasegawa M, Smith MJ, Crowther RA (1996) Assembly of microtubule-associated protein tau into Alzheimer-like filaments induced by sulphated glycosaminoglycans. Nature 383:550-553

20. Goedert M, Spillantini MG, Cairns NJ, Crowther RA (1992) Tau proteins of Alzheimer paired helical filaments: abnormal phosphorylation of all six brain isoforms. Neuron 8:159-168

21. Goedert M, Spillantini MG, Jakes R, Rutherford D, Crowther RA (1989) Multiple isoforms of human microtubule-associated protein tau: sequences and localization in neurofibrillary tangles of Alzheimer's disease. Neuron 3:519-526

22. Goedert M, Wischik CM, Crowther RA, Walker JE, Klug A (1988) Cloning and sequencing of the cDNA encoding a core protein of the paired helical filament of Alzheimer disease: identification as the microtubule-associated protein tau. Proc Natl Acad Sci (USA) 85:4051-4055

23. Guo JL, Lee VM (2014) Cell-to-cell transmission of pathogenic proteins in neurodegenerative diseases. Nat Med 20:130-138

24. He S, Scheres SHW (2017) Helical reconstruction in RELION. J Struct Biol 198:163-176

25. Jackson SJ, Kerridge C, Cooper J, Cavallini A, Falcon B, Cella CV, Landi A, Szekeres PG, Murray TK, Ahmed Z et al (2016) Short fibrils constitute the major species of seed-competent tau in the brains of mice transgenic for human P301S tau. J Neurosci 36:762-772

26. Levy-Lahad E, Wasco W, Poorkaj P, Romano DM, Oshima J, Pettingell WH, Yu CE, Jondro PD, Schmidt SD, Wang K et al (1995) Candidate gene for the chromosome 1 familial Alzheimer's disease locus. Science 269:973-977

27. Masters CL, Simms G, Weinman NA, Multhaup G, McDonald BL, Beyreuther K (1985) Amyloid plaque core protein in Alzheimer disease and Down syndrome. Proc Natl Acad Sci (USA) 82:4245-4249

28. Mudher A, Colin M, Dujardin S, Medina M, Dewachter I, Alavi Naini SM, Mandelkow EM, Mandelkow E, Buée L, Goedert $M$ et al (2017) What is the evidence that tau pathology spreads through prion-like propagation? Acta Neuropathol Commun 5:99
29. Murrell J, Farlow M, Ghetti B, Benson MD (1991) A mutation in the amyloid precursor protein associated with hereditary Alzheimer's disease. Science 254:97-99

30. Murshudov GN, Vagin AA, Dodson EJ (1997) Refinement of macromolecular structures by the maximum-likelihood method. Acta Crystallogr D 53:240-255

31. Pérez M, Valpuesta JM, Medina M, Montejo de Garcini E, Avila J (1996) Polymerization of tau into filaments in the presence of heparin: the minimal sequence required for tau-tau interaction. $\mathrm{J}$ Neurochem 67:1183-1190

32. Rasmussen J, Mahler J, Beschorner N, Kaeser SA, Hasler LM, Baumann F, Nyström S, Portelius E, Blennow K, Lashley T et al (2017) Amyloid polymorphisms constitute distinct clouds of conformational variants in different etiological subtypes of Alzheimer's disease. Proc Natl Acad Sci (USA) 114:13018-13023

33. Scheltens P, Blennow K, Breteler MM, de Strooper B, Frisoni GB, Salloway S, Van der Flier WM (2016) Alzheimer's disease. Lancet 388:505-517

34. Scheres SH (2012) RELION: implementation of a Bayesian approach to cryo-EM structure determination. J Struct Biol 180:519-530

35. Sherrington R, Rogaev EI, Liang Y, Rogaeva EA, Levesque G, Ikeda M, Chi H, Lin C, Li G, Holman K et al (1995) Cloning of a gene bearing missense mutations in early-onset familial Alzheimer's disease. Nature 375:754-760

36. Spina S, Farlow MR, Unverzagt FW, Kareken DA, Murrell JR, Fraser G, Epperson F, Crowther RA, Spillantini MG, Goedert M et al (2008) The tauopathy associated with mutation +3 in intron 10 of Tau: characterization of the MSTD family. Brain 131:72-89

37. Strittmatter WJ, Saunders AM, Schmechel D, Pericak-Vance M, Enghild J, Salvesen GS, Roses AD (1993) Apolipoprotein E: highavidity binding to beta-amyloid and increased frequency of type 4 allele in late-onset familial Alzheimer disease. Proc Natl Acad Sci (USA) 90:1977-1981

38. Taniguchi-Watanabe S, Arai T, Kametani F, Nonaka T, Masuda-Suzukake M, Tarutani A, Murayama S, Saito Y, Arima K, Yoshida $\mathrm{M}$ et al (2016) Biochemical classification of tauopathies by immunoblot, protein sequence and mass spectrometric analyses of sarkosyl-insoluble and trypsin-resistant tau. Acta Neuropathol 131:267-280

39. Tycko R (2015) Amyloid polymorphism: structural basis and neurobiological relevance. Neuron 86:632-645

40. Wang Y, Mandelkow E (2016) Tau in physiology and pathology. Nat Rev Neurosci 17:5-21

41. Wischik CM, Novak M, Edwards PC, Klug A, Tichelaar W, Crowther RA (1988) Structural characterization of the core of the paired helical filament of Alzheimer disease. Proc Natl Acad Sci (USA) 85:4884-4888

42. Wischik CM, Novak M, Thøgersen HC, Edwards PC, Runswick MJ, Jakes R, Walker JE, Milstein C, Roth M, Klug A (1988) Isolation of a fragment of tau derived from the core of the paired helical filament of Alzheimer disease. Proc Natl Acad Sci (USA) 85:4506-4510

43. Zhang K (2016) Gctf: Real-time CTF determination and correction. J Struct Biol 193:1-12

44. Zheng SQ, Palovcak E, Armache JP, Verba KA, Cheng Y, Agard DA (2017) MotionCor2: anisotropic correction of beaminduced motion for improved cryo-electron microscopy. Nat Meth $14: 331-332$ 